\title{
FATORES ASSOCIADOS À ADESÃO AO USO DE PALMILHAS BIOMECÂNICAS
}

\author{
Guimarães CQ ${ }^{1}$, Teixeira-Salmela LF ${ }^{2}$, Rocha IC ${ }^{1}$, Bicalho LI ${ }^{1}$ e Sabino GS ${ }^{1}$ \\ ${ }^{1}$ Universidade Federal de Minas Gerais - UFMG, Belo Horizonte, MG - Brasil \\ ${ }^{2}$ Departamento de Fisioterapia, Escola de Educação Física, Fisioterapia e Terapia Ocupacional, UFMG Belo Horizonte, \\ MG - Brasil \\ Correspondência para: Professora Luci Fuscaldi Teixeira - Salmela, Ph.D., Departamento de Fisioterapia , UFMG, Av. \\ Antonio Carlos, 6627, Campus Pampulha, CEP 31270-901, Belo Horizonte, MG - Brasil, e-mail: lfts@ufmg.br
}

Recebido: 20/04/2005 - Aceito: 08/03/2006

\begin{abstract}
RESUMO
Contextualização: Os benefícios das palmilhas biomecânicas no tratamento de acometimentos nos membros inferiores têm sido relatados. No entanto, observa-se na prática uma baixa adesão ao uso dessa órtese, comprometendo os benefícios que poderiam ser proporcionados. Objetivo: Investigar os fatores relacionados à adesão ao uso de palmilhas biomecânicas. Método: Participaram deste estudo 33 indivíduos (43,97 \pm 14,73 anos) para os quais foram confeccionadas palmilhas biomecânicas. Um questionário contendo questões a respeito da palmilha e seu uso foi aplicado, em forma de entrevista, sendo as variáveis de estudo estabelecidas a partir dessas questões. Com base nos resultados obtidos, os indivíduos foram separados em 4 grupos, de acordo com o nível de adesão: adesão total, adesão parcial, adesão inconstante e não-adesão. Utilizou-se o teste "KruskalWallis” para investigar se esses grupos diferiam entre si em relação às variáveis pesquisadas. Coeficientes de Correlação de Spearman foram utilizados para verificar possíveis correlações entre essas variáveis e adesão. Resultados: Dos 33 participantes, $15,2 \%$ tiveram adesão total, enquanto 42,4\% abandonaram a palmilha. Comparando-se os 4 grupos de adesão em relação às variáveis pesquisadas, observou-se diferença significativa para conforto da palmilha $(p=0,003)$, grau de melhora atribuído à palmilha $(p=0,006)$ e grau de conhecimento sobre o problema $(p<0,002)$. Correlações significativas $\left(r_{s}=0,58 ; p<0,001\right.$ e $r_{s}=0,50$; $p<0,01$ ) foram observadas entre adesão e conforto e grau de melhora atribuído à palmilha, respectivamente. Conclusão: Resultados do presente estudo revelaram que conforto e grau de melhora atribuído à palmilha podem influenciar na adesão ao uso dessas órteses, constituindo-se fatores importantes para o sucesso do tratamento.
\end{abstract}

Palavras-chave: palmilhas biomecânicas, adesão, órtese.

\section{ABSTRACT}

\section{Factors Related to Compliance with Biomechanical Insole Use}

Background: The benefits of biomechanical insoles for treating lower limb impairment have been reported. However, in clinical practice, low compliance with the use of such orthoses has been observed, which detracts from the possible benefits. Objective: To investigate the factors related to compliance with biomechanical insole use. Method: Thirty-three subjects (aged $44 \pm 14.7$ years) for whom biomechanical insoles had been molded took part in this study. A questionnaire asking about the insoles and their use was applied through interviews. The study variables were established from these questions. Based on the results obtained, the participants were divided into four groups according to their compliance level: full compliance, partial compliance, irregular compliance and non-compliance. The Kruskal-Wallis test was utilized to assess differences between these groups regarding the variables investigated. Spearman correlation coefficients were utilized to investigate possible relationships between these variables and the compliance. Results: Among the 33 participants, 15.2\% were fully compliant, while $42.4 \%$ abandoned the insoles. Comparing the four compliance groups in relation to the variables investigated, significant differences were observed regarding insole comfort ( $p=0.003)$, improvement attributed to the insoles $(p=0.006)$, and degree of knowledge of the problem $(p<0.002)$. Significant correlations $\left(r_{s}=0.58 ; p<0.001\right.$; and $\left.r_{s}=0.50 ; p<0.01\right)$ were observed between compliance and the comfort and improvement attributed to the insoles, respectively. Conclusion: The findings from this study revealed that the comfort and degree of improvement attributed to the insoles may influence the compliance with the use of these orthoses. These factors are important for the success of the treatment.

Key words: biomechanical insoles, compliance, orthosis. 


\section{INTRODUÇÃO}

A adesão, essencial para o sucesso de um tratamento ${ }^{1}$, pode ser definida como o correto cumprimento às prescrições terapêuticas e geralmente requer uma mudança sustentada de comportamento por parte do indivíduo². Quando a adesão não ocorre, há uma redução considerável dos benefícios que poderiam ser gerados, seja qual for a terapia implemen$\operatorname{tada}^{1,2,3}$.

Considerando a adesão de forma genérica, relatam-se vários fatores que podem influenciar o cumprimento das prescrições terapêuticas por parte do paciente: sua idade, escolaridade, a gravidade de sua condição $0^{4} \mathrm{e}$ a forma como ele a percebe ${ }^{3,4}$, o impacto da deficiência sobre sua vida ${ }^{5}$, seu nível de motivação ${ }^{4,6}$, a forma como as orientações lhe são transmitidas (se oralmente ou por escrito) ${ }^{7}$, suas experiências com outros tratamentos, sua percepção sobre quem é o responsável pela sua melhora ${ }^{8}$, o esclarecimento sobre sua condição ${ }^{9}$, o incentivo recebido do terapeuta ${ }^{10,11} \mathrm{e}$ a duração do tratamento ${ }^{5}$. Quando o tratamento envolve a prescrição de uma órtese, foi observado que o conforto, a fácil utilização, a relevância de seu uso durante a realização das atividades diárias e sua propriedade em não realçar as deficiências do paciente constituem pontos básicos para sua aceitação ${ }^{11}$.

Palmilhas biomecânicas são órteses fabricadas com material termomoldável (E.V.A) e são utilizadas no interior de calçados de indivíduos com alterações estruturais no pé. Essas alterações podem originar compensações durante atividades funcionais como marcha, corrida e práticas esportivas, levando a disfunções e patologias ${ }^{12,13}$. O uso das palmilhas visa acomodar tais alterações, controlando assim o movimento de pronação excessiva na articulação subtalar ${ }^{14}$ e contribuindo para reduzir a dor, evitar a progressão ou desenvolvimento de morbidades e melhorar a capacidade funcional do paciente $e^{13,15,16,17}$.

Além desses efeitos positivos, já foi demonstrado que as palmilhas biomecânicas possuem boa relação custobenefício, podendo ser usadas como primeira opção para o tratamento de síndrome patelofemoral de grau moderado a severo ${ }^{17}$. Apesar disso, observa-se na prática uma baixa adesão a esse tipo de tratamento. A não-adesão a uma terapia, em adição à redução dos benefícios esperados, poderá causar considerável frustração ao paciente e ao terapeuta ${ }^{3}$ e acarretar grandes custos econômicos ${ }^{3,19,20}$. O aumento dos custos será conseqüência do maior tempo de afastamento das atividades ou da necessidade de buscar uma nova intervenção.

Estima-se que $60 \%$ dos pacientes que sofrem de acometimentos crônicos não seguem adequadamente o tratamento proposto ${ }^{19}$. Compreender os fatores relacionados à baixa adesão ao uso de palmilhas, por exemplo, pode auxiliar na seleção de estratégias adequadas à melhoria de sua aceitação. O que se observa, no entanto, é que a literatura é escassa com relação a estudos que investigaram tais correlações. Desse modo, o objetivo deste trabalho foi investigar os fatores relacionados à adesão ao uso de palmilhas biomecânicas como forma terapêutica.

\section{METODOLOGIA}

\section{Participantes}

Participaram deste estudo 33 indivíduos, para os quais foram confeccionadas palmilhas biomecânicas. Os seguintes critérios de inclusão foram aplicados: ter sido a palmilha indicada para acomodação de alguma alteração biomecânica nos pés; terem se passado de 25 a 30 meses após a confecção da palmilha; estar na faixa etária entre 18 e 65 anos; residir ou trabalhar na região metropolitana de Belo Horizonte e ter recebido orientação para fazer uso diário da palmilha. O critério de exclusão considerado foi: ter o paciente sofrido qualquer intercorrência que impossibilitasse o uso da palmilha.

\section{Instrumentação e procedimentos}

De acordo com os critérios de inclusão estabelecidos, 142 indivíduos foram inicialmente selecionados a partir do cadastro de pacientes da clínica. Eles foram então contactados por telefone para esclarecimentos sobre o estudo e, havendo interesse de participação, foi agendada uma entrevista, a ser realizada por um dos pesquisadores em local e horário de preferência. Antes de responder à entrevista, foi solicitado a cada indivíduo que lesse e assinasse o termo de consentimento livre e esclarecido, aprovado pelo Comitê de Ética em Pesquisa da Universidade Federal de Minas Gerais (ETIC 05/04), concordando com sua participação no estudo.

Os dados foram obtidos através da aplicação de um questionário composto por questões objetivas e subjetivas a respeito da palmilha e do seu uso, além de caracterizar os indivíduos com relação a variáveis sócio-demográficas e clínicas (Anexo 1).

A seleção das questões foi feita com base nos fatores apontados pela literatura como importantes para se determinar a adesão do paciente aos diferentes regimes terapêuticos, como características demográficas ${ }^{4}$, características do problema / disfunção ${ }^{4}$, percepções do usuário ${ }^{3,4,5,8}$ e suas experiências com outros tratamentos ${ }^{8}$. Com base nesses fatores, as variáveis do estudo foram definidas (Tabela 1).

Para verificar a necessidade de adequação das perguntas do questionário foi realizado um estudo piloto com 5 indivíduos que faziam uso de palmilhas. Não foi necessária nenhuma modificação no questionário.

Com base nos resultados obtidos, os indivíduos foram agrupados em categorias, levando-se em consideração o nível de adesão:

- Adesão total (Grupo 1) - indivíduos que seguem corretamente as orientações terapêuticas, fazendo uso diário da palmilha por no mínimo 8 horas;

- Adesão parcial (Grupo 2) - indivíduos que usam a palmilha diariamente, mas por período inferior ao orientado; 


\section{ANEXO 1: Questionário}

\begin{tabular}{|lll}
\hline Nome: $\_$Telefone: \\
Sexo $\square$ Masculino $\square$ Feminino & Idade: $\_$Data de Nascimento: \\
Escolaridade: & ID: $\quad$ Ocupação: $\_$
\end{tabular}

1. Que problema o levou a colocar a palmilha?

2. Como você considera o seu grau de conhecimento sobre esse problema?

- Possuo grande conhecimento • Razoável conhecimento • Pouco conhecimento

3. Com relação à gravidade desse problema, você acredita que ele seja (sendo 0 o grau mais leve possível e 10 aquele com maior gravidade)

4. Quais tipos de atividades foram alteradas devido a esse problema (antes de colocar a palmilha)?

- Esportiva/Recreativa • Atividades de vida diária • Ocupacionais • Nenhuma

5. Você realizou algum outro tratamento para esse problema?

- Sim. Antes de colocar a palmilha. Qual:

- Sim. Simultaneamente ao uso da palmilha. Qual:

- Não

6. Qual foi a orientação passada em relação ao tempo diário de uso da palmilha?

7. De que forma a orientação sobre uso da palmilha foi passada?

- Recebi orientação oral • Recebi orientação escrita e oral

8. Você ainda usa a palmilha?

- Sim - exatamente como foi indicado (mínimo de 8 horas por dia) • Sim - eventualmente

- Sim - diariamente, mas por tempo menor do que indicado

- Não. Por quanto tempo você usou?

Para resposta negativa: Por que não usa?

Para resposta positiva: Nos momentos em que não usa sente alguma diferença?

- Sim. Qual?__ Não

9. Você considera que a palmilha melhorou seu problema (sendo 0 se não houve melhora e 10 melhora total)

10. Você sabe como a palmilha atua para melhorar o seu problema?

• Não sei • • Sei um pouco • Sei bastante.

11. Quanto ao conforto, você considera a palmilha (sendo 0 o mais desconfortável e 10 o mais confortável possível)

12. Quanto ao preço, você considera a palmilha:

- Cara - Acessível B Barata

13. Você ficou satisfeito com o terapeuta que indicou a palmilha (sendo 0 a menor satisfação possível e 10 a satisfação máxima):

14. Você ficou satisfeito com o terapeuta que colocou a palmilha (sendo 0 a menor satisfação possível e 10 a satisfação máxima):

15. Qual tipo de calçado você mais usa?

- Sandália $\quad$ • Tênis $\quad$ - Sapato $\quad$ Chinelo Sapato ou sandália de salto alto

16. A palmilha cabe em todos os calçados “fechados”?

- Sim • Não. Em quais?

17. Você é: • • Sedentário • Moderadamente ativo (menos que 3 vezes por semana) • Ativo

18. De quem é a principal responsabilidade pela melhora de sua condição de saúde? 
- Adesão inconstante (Grupo 3) - indivíduos que usam a palmilha apenas eventualmente: aqueles que, por exemplo, fazem uso descontínuo ou que fazem uso apenas durante a realização de atividades esportivas ou de lazer;

• Não-adesão (Grupo 4) - indivíduos que não usam mais a palmilha.

\section{Análise estatística}

O programa SPSS para Windows (versão 10.07) foi utilizado para análise. Estatísticas descritivas e análises de freqüência foram efetuadas para todas as variáveis. Utilizouse o teste "Kruskal-Wallis" para investigar se os grupos nãoadesão, pobre adesão, moderada adesão e adesão total diferiam entre si em relação às variáveis estudadas. Para as variáveis em que houve diferença significativa, foram verificadas as correlações com a adesão, utilizando-se o Coeficiente de Correlação de Spearman.

\section{RESULTADOS}

\section{Caracterização da amostra}

Do total de 142 indivíduos cadastrados, 109 não participaram do estudo, pelos seguintes motivos: mudança do número de telefone (53), não atendimento às chamadas telefônicas (25), cancelamento repetido da entrevista agendada (4), impossibilidade de conversar diretamente com o usuário da palmilha, apesar de ter sido estabelecido contato telefônico com parentes e/ou colegas de trabalho (17), desinteresse na participação no estudo (10).

Dessa forma, participaram do estudo 33 indivíduos, sendo 14 homens (42,4\%) e 19 mulheres (57,6\%), com média de idade de 43,97 ( \pm 14,73) anos (variação de 19 - 63 anos). Dor no tornozelo/pé e no joelho foram os problemas mais freqüentes (57,6\%). Dezesseis indivíduos (48,5\%) possuíam curso superior completo e 25 (75,8\%) exerciam atividade remunerada. As características da amostra estão apresentadas na Tabela 2.

\section{Adesão ao tratamento}

Dos 33 participantes, cinco (15,2\%) foram caracterizados como adesão total, cinco (15,2\%) como adesão parcial, nove (27,3\%) como adesão inconstante e 14 $(42,4 \%)$ como não-adesão. Para o grupo não-adesão, o tempo médio de uso da palmilha antes de abandoná-la foi 193,36 dias (2 - 730 dias).

\section{Motivos apontados para a não-adesão}

Para os 14 participantes que abandonaram o uso da palmilha, foi perguntada a razão para a não-adesão. Em suas respostas, os participantes freqüentemente relataram mais de um motivo. As razões apontadas foram: "dificuldade da palmilha caber nos sapatos” (42,1\%); “desconforto” (21,4\%); “dor” (21,4\%); “não houve melhoras” (21,4\%); “dificuldade para adaptação à palmilha” (14,3\%); “não gostar de usar sapato fechado" (14,3\%); “trabalho não permite uso de calçado que caiba a palmilha” (7,1\%), "palmilha não cabe em nenhum calçado” (7,1\%) e “preço elevado” (7,1\%).

Quando questionados se a palmilha cabia em todos os sapatos fechados, mais de $80 \%$ dos participantes responderam negativamente, entre os quais $75 \%$ apontaram que ela só cabia em tênis. Foi então analisado se o tipo de calçado no qual a palmilha se encaixava adequadamente correspondia ao calçado que a pessoa mais usava. No grupo adesão total, isso ocorreu com todos os indivíduos, enquanto nos demais grupos ocorreram, em média, com 41\% dos participantes.

\section{Relações entre adesão e variáveis estudadas}

Comparando-se os grupos não-adesão, adesão inconstante, adesão parcial e adesão total em relação às variáveis estudadas, o teste Kruskal-Wallis evidenciou diferenças sig-

Tabela 1. Definição das Variáveis do Estudo a partir dos Fatores Observados como importantes para a Adesão a Diferentes Regimes Terapêuticos.

\section{$\begin{array}{ll}\text { FATOR OBSERVADO } & \text { VARIÁVEL ESTUDADA }\end{array}$}

\section{Características demográficas}

Características do problema

Percepções do usuário

Tratamentos realizados para o problema / disfunção

Forma de orientação para uso da palmilha

Calçados

Atividade física
Sexo; idade; escolaridade; ocupação

Problema/disfunção; atividades alteradas

Grau de conhecimento do problema; grau de conhecimento sobre o mecanismo de ação da palmilha; gravidade que o problema representa; grau de melhora atribuído à palmilha; preço da palmilha; conforto da palmilha; grau de satisfação com o profissional que confeccionou a palmilha; pessoa a quem o usuário atribui a principal responsabilidade da manutenção de sua saúde

Ausência/presença (concomitante ou anterior) de tratamentos associados ao uso da palmilha

Orientação verbal e/ou escrita

Tipo de calçado mais utilizado pelo usuário; Capacidade de adaptação da palmilha aos diversos calçados; correspondência entre tipo de calçado que cabe a palmilha e o tipo mais utilizado pelo usuário

Nível de atividade física do usuário 
nificativas para conforto $(p<0,003)$, grau de melhora atribuído à palmilha $(p<0,006)$ e grau de conhecimento do problema $(p<0,002)$. A Tabela 3 apresenta os níveis de significância para todas as variáveis analisadas.

Coeficientes de correlação de Spearman, calculados para essas 3 variáveis, revelaram que apenas conforto e grau de melhora atribuído à palmilha se correlacionaram significativamente com o nível de adesão, apresentando coeficientes de correlação de 0,58 e 0,50, respectivamente.

Não foi observada correlação significativa entre o grau de conhecimento do problema e a adesão. Os indivíduos do grupo adesão inconstante apresentaram percepção de conhecimento significativamente maior que indivíduos dos grupos adesão total e não-adesão.

\section{DISCUSSÃO}

O presente estudo teve como objetivo investigar os fatores relacionados à adesão ao uso de palmilhas biomecânicas. O termo adesão refere-se à exatidão com que o indivíduo segue as orientações terapêuticas ${ }^{2}$, devendo ser definido com parâmetros apropriados ao regime terapêutico ou comportamento de saúde sob estudo ${ }^{20}$. No caso das palmilhas, não há uma orientação específica sobre a quantidade de horas

Tabela 2. Caracterização da Amostra (n=33).

\begin{tabular}{lclc}
\hline \multicolumn{3}{c}{ VARIÁVEL } \\
\hline $\begin{array}{l}\text { Sexo } \\
\text { Feminino } \\
\text { Masculino }\end{array}$ & $19(57,6 \%)$ & $\begin{array}{l}\text { Dor no tornozelo/pé } \\
\text { Dor no joelho }\end{array}$ & $13(39,4 \%)$ \\
Idade (anos) & $14(42,4 \%)$ & $6(18,2 \%)$ \\
$\quad$ Média \pm DP & $43,97 \pm 14,73$ & Dor na coluna & $3(9,1 \%)$ \\
Variação & $19-64$ & Dor em mais de um local & $3(9,1 \%)$ \\
Escolaridade & & Alterações na marcha & $2(6,1 \%)$ \\
$\quad$ Superior completo & $16(48,5 \%)$ & Discrepância de MMII & $2(6,1 \%)$ \\
$\quad$ Superior incompleto & $7(21,2 \%)$ & Dor no quadril & $1(3 \%)$ \\
Ensino médio & $6(18,2 \%)$ & Ocupação & \\
Ensino fundamental & $3(9,1 \%)$ & Atividade remunerada & $25(75,8 \%)$ \\
Pós-graduação & $1(3 \%)$ & Aposentado & $4(12,1 \%)$ \\
& & Sem atividade remunerada & $4(12,1 \%)$ \\
\hline
\end{tabular}

Tabela 3. Comparação dos grupos com relação às variáveis investigadas.

\begin{tabular}{lcc}
\hline \multicolumn{1}{c}{ Variável } & Valor Crítico & Valor de $\boldsymbol{p}$ \\
\hline Grau de satisfação com o profissional que indicou a palmilha & 1,305 & 0,73 \\
Grau de satisfação com terapeuta que confeccionou a palmilha & 3,886 & 0,27 \\
Gravidade que o problema representa & 4,192 & 0,24 \\
Nível de atividade física do usuário & 1,68 & 0,64 \\
Capacidade de adaptação da palmilha aos diversos calçados & 0,276 & 0,96 \\
Grau de melhora atribuído à palmilha & 12,427 & $0,006^{*}$ \\
Conforto da palmilha & 13,791 & $0,003^{*}$ \\
Escolaridade & 2,205 & 0,53 \\
Ocupação (por área) & 1,89 & 0,60 \\
Preço da palmilha & 1,209 & 0,75 \\
Grau de conhecimento sobre o problema & 14,995 & $0,002^{*}$ \\
Problema/disfunção & 1,962 & 0,58 \\
Grau de conhecimento sobre o mecanismo de ação da palmilha & 3,758 & 0,29 \\
Correspondência entre tipo de calçado que cabe a palmilha e tipo & 6,491 & 0,09 \\
de calçado mais utilizado pelo usuário & & \\
\hline
\end{tabular}


de uso diário (pacientes são orientados a usar a palmilha pelo máximo de tempo possível), tornando difícil uma classificação precisa do nível de adesão. Neste estudo, considerou-se como adesão total o uso da palmilha por no mínimo 8 horas/dia, tomando como base o período de tempo diário que o indivíduo realizaria atividades fora de casa, como trabalho, estudo, lazer, etc.

As variáveis estudadas foram definidas a partir dos fatores apontados na literatura como importantes para a adesão a diferentes regimes terapêuticos. O tipo de calçado mais utilizado pelo usuário foi investigado, apesar da falta de embasamento na literatura, por ter sido considerado relevante pelos pesquisadores. Acredita-se que ele poderia influenciar na adesão, já que alguns modelos inviabilizam a colocação da palmilha. Os dados obtidos nesse estudo não apontaram correlação significativa entre adesão e a variável “correspondência entre tipo de calçado que cabe a palmilha e o tipo mais utilizado pelo usuário”. No entanto, todas as pessoas que tiveram adesão total relataram que a palmilha se adaptava bem ao calçado que mais utilizavam, enquanto nos demais grupos isso ocorreu em média com $41 \%$ dos pacientes. Entre as pessoas que abandonaram o tratamento, alguns motivos apontados foram: o fato da palmilha não se adaptar em sapatos, apenas ao tênis; desconforto; e incompatibilidade entre o uso do calçado que se adapta à palmilha e seu trabalho. A perspectiva de ter que aderir ao uso de uma órtese que limita as opções de calçados, gerando a necessidade de mudança no estilo de vida (que pode ser conflitante com compromissos sociais ou de trabalho) pode ter contribuído para uma diminuição da adesão.

Foi verificada significativa correlação entre a adesão ao tratamento e o conforto proporcionado pela palmilha ( $r s=0,58 ; p<0,001)$. Basford et al. ${ }^{11}$ investigaram fatores que poderiam contribuir para a aceitação de uma órtese para membro superior e concluíram que, mesmo produzindo efeitos benéficos, o uso da órtese é descartado se ela for desconfortável. Como demonstrado em estudos prévios ${ }^{21,22}$, os indivíduos podem ter experimentado algum fator gerador de desconforto, levando a não-adesão, tais como: fatores intrínsecos da palmilha (comprimento e/ou largura incorretos e áreas de grande compressão no arco do pé) ou fatores biomecânicos (excesso de trabalho muscular para conter vibrações decorrentes do impacto ou para proporcionar estabilização - gerando fadiga). Segundo relatos dos pacientes, um fator importante gerador de desconforto refere-se ao grande volume ocupado pela palmilha dentro do calçado, deixando-o apertado.

Correlação significativa foi também observada entre a adesão e o grau de melhora atribuído à palmilha. A relação causa-efeito é difícil de ser definida, por se tratar de uma investigação retrospectiva. Na fase inicial do tratamento, quando o paciente está sintomático, o alívio dos sintomas promovido pelo uso da palmilha pode ter funcionado como reforço positivo para a sua adesão. Além disso, nessa fase, é maior o contato com o terapeuta que confeccionou a palmilha (para realização de ajustes) e com o terapeuta que indicou a órtese (quando faz parte do programa de reabilitação). Como demonstrado em estudos prévios, parte da adesão é baseada na relação terapeuta-paciente e os resultados dependem do interesse e envolvimento de ambos $^{5,23}$. Por outro lado, pode-se inferir também a relação causa-efeito inversa. Vários estudos estabeleceram que os efeitos de determinado tratamento dependem em parte da adesão do paciente, de forma que a não-adesão reduz a eficácia do mesmo ${ }^{2,3}$. Dessa forma, a diminuição dos sintomas pode ter sido conseqüência da correta adesão do indivíduo ao tratamento.

Uma vez que se opte pela palmilha como parte integrante do tratamento, a sua utilização poderá acompanhar o indivíduo por vários anos. Dessa forma, torna-se necessária uma adesão em longo prazo. Vários estudos demonstram que quanto mais longo um regime terapêutico, menor a possibilidade de adesão ${ }^{1,3,5,6}$. Alguns componentes importantes para a adesão na fase inicial não estão mais presentes, como a perspectiva de remissão ou alívio dos sintomas (pois de fato isso já pode ter ocorrido), ou o contato freqüente com os terapeutas. Fazse necessário encarar o tratamento sob uma nova ótica. Seu objetivo deixa de ser a cura, passando a ser a prevenção, que, na sua forma ideal, é direcionada ao infinito, significando que o produto do tratamento de fato não é alcançado, e o objetivo na sua essência é um processo ao invés de um produto $^{24}$. Assim, entender os conceitos que definem prevenção é uma premissa importante para tornar mais efetiva à adesão a tratamentos mais longos.

Um outro aspecto a ser considerado é que tratamentos em longo prazo e preventivos geralmente requerem uma maior educação do paciente. Essa educação inclui todos os esforços e processos para dar informação terapêutica ou modificar o comportamento e crenças em relação aos cuidados com a saúde. É reportado que pacientes com maior esclarecimento sobre sua condição tendem a apresentar maior adesão ${ }^{9}$. No entanto, os achados do presente estudo demonstraram que indivíduos do grupo adesão inconstante possuem conhecimento significativamente maior que indivíduos dos grupos adesão total e não-adesão. A ausência de correlação entre o grau de conhecimento do problema e a adesão ao uso da palmilha causou surpresa. Um importante aspecto a ser considerado é que esse estudo pesquisou a percepção do usuário em relação ao seu conhecimento sobre o problema, o que pode não corresponder ao conhecimento real, requerendo cautela na interpretação desses resultados. Além disso, os estudos que relataram a importância do conhecimento investigaram a adesão à medicação prescrita ${ }^{9,23}$, que pode ser bem diferente da adesão ao uso de uma órtese, onde aspectos como conforto e grau de melhora do problema parecem ser mais relevantes. 


\section{CONCLUSÃO}

Os achados do presente estudo revelaram que o conforto e grau de melhora atribuído à palmilha influenciaram a adesão ao uso das palmilhas biomecânicas. Sendo assim, esses fatores devem ser considerados durante a indicação dessas órteses, de forma a otimizar os resultados do tratamento.

\section{REFERÊNCIAS BIBLIOGRÁFICAS}

1. Oldridge NB. Cardiac rehabilitation exercise programme - Compliance and compliance-enhancing strategies. Sports Med. 2003 June; 6:42-55.

2. Agras WS. Understanding compliance with the medical regimen: The scope of the problem and a theoretical perspective. Arthritis Care Res. 1989 Sept; 2 (3 Suppl): S2-S7.

3. Faleiros JJ. Os pacientes seguem adequadamente os procedimentos terapêuticos? Prát Méd Geral. 1982 Feb; 26(2):120-3.

4. Sluijs EM, Kok GJ, Van der ZJ. Correlates of exercise compliance in physical therapy. Phys Ther. 1993 Nov; 73 (11): 771-82.

5. Dimou C. Patient education. Part III. Patient compliance. Dis Mon. 2000 Dec; 46 (12): 811-22.

6. Friedrich M, Gittler G, Halberstadt Y, Cermak T, Heiller I. Combined exercise and motivation program: effect on the compliance and level of disability of patients with chronic low back pain: a randomized controlled trial. Arch Phys Med Rehabil. 1998 May; 79 (5): 475-87.

7. Takala J, Niemela N, Rosti J, Sievers K. Improving compliance with therapeutic regimens in hypertensive patients in a community health center. Circulation. 1979 Mar; 59 (3): 540-3.

8. Kurita GP, Andrucioli MP. Adesão ao tratamento da dor crônica: Estudo de variáveis demográficas, terapêuticas e psicossociais. Arq Neuropsiquiatr. 2003 Jul; 61 (2-B): 416-25.

9. Dent T. Part I. Patient education. Dis Mon. 2000 Dec; 46 (12): 785-97.

10. Cott C, Finch E. Goal-setting in physical therapy practice. Physiother Can. 1991 Jan; 43 (1): 19-22.

11. Basford JR, Johnson SJ. Form may be as important as function in orthotic acceptance: a case report. Arch Phys Med Rehabil. 2002 Mar; 83 (3): 433-5.

12. Hoffman S, Peterson MR. Foot orthotics: An overview of rationale, assessment and fabrications. 2001; 3 (25): 509-26.
13. Prescription Custom Foot Orthoses Practice Guidelines (Database on the internet). Ellicott City: The American College of Foot and Ankle Orthopedics \& Medicine. 2002. Avaible from: http://www.acfaom.org/pg1103.pdf

14. Johanson MA, Donatelli R, Wooden MJ, Andrew PD, Cummings GS. Effects of Three Different Posting Methods on Controlling Abnormal Subtalar Pronation. Phys Ther. 1994 Feb; 74 (2): 149-161.

15. Gross MT, Byers JM, Krafft JL, Lackey EJ, Melton KM. The impact of custom semirigid foot orthotics on pain and disability for individuals with plantar fasciitis. J Orthop Sports Phys Ther. 2002 Apr; 32 (4): 149-57.

16. Amelp TK, Stothart JP, Kumar S. The effectiveness of funcional foot orthoses as a treatment for patellofemoral stress syndrome: The clients' perspective. Physiother Can. 2000; 52 (2): 153-7.

17. Piman D, Jack DA. Clinical investigation to determine the effectiveness of biomechanical foot orthoses as initial treatment for patellofemoral syndrome. J Prosth Orth. 2000; 12 (4): 111-8.

18. Bird AR, Bendrups AP, Payne CB. The effect of foot wedging on electromyographic activity in the erector spinae and gluteus medius muscles during walking. Gait Posture. 2003 Oct; 18 (2): 81-91.

19. Dunbar-Jacob J, Mortimer-Stephens MK. Treatment adherence in chronic disease. J Clin Epidemiol. 2001 Dec; 54 (1 Suppl): S57-60.

20. Vitolins MZ, Rand CS, Rapp SR, Ribisl PM, Sevick MA. Measuring adherence to behavioral and medical interventions. Control Clin Trials. 2000 Oct; 21 (5 Suppl): S188-S94.

21. Vicenzino B. Foot orthotics in the treatment of lower limb conditions: a musculoskeletal physiotherapy perspective. Man Ther. 2004 Nov; 9 (4): 185-96.

22. Nigg BM, Nurse MA, Stefanyshyn DJ. Shoe inserts and orthotics for sport and physical activities. Med Sci Sports Exerc. 1999 July; 31 (7 Suppl): S421-8.

23. Gomes MM, Eetefan IJS. Reforçar a aderência à medicação prescrita? Aspectos práticos. Arq Bras Med. 1992; 66 (1): 414.

24. Sluijs EM, Knibbe JJ. Patient compliance with exercises: Different theoretical approaches to short-term and long-term compliance. Patient Edu Couns. 1991; 17: 191-204. 